\title{
Effect of Dietary Intake of Avocado Oil and Olive Oil on Biochemical Markers of Liver Function in Sucrose-Fed Rats
}

\author{
Octavio Carvajal-Zarrabal, ${ }^{1}$ Cirilo Nolasco-Hipolito, \\ ${ }^{2}$ Ma. Guadalupe Aguilar-Uscanga, ${ }^{3}$ Guadalupe Melo Santiesteban, ${ }^{4}$ \\ Patricia M. Hayward-Jones, ${ }^{1}$ and Dulce Ma. Barradas-Dermitz ${ }^{5}$ \\ ${ }^{1}$ Biochemical and Nutrition Chemistry Area, University of Veracruz, SS Juan Pablo II $s / n$, \\ 94294 Boca del Río, VER., Mexico \\ ${ }^{2}$ Department of Molecular Biology, Faculty of Resource Science and Technology, Universiti Malaysia Sarawak, Kota Samarahan, \\ 94300 Sarawak, Malaysia \\ ${ }^{3}$ Food Research and Development Unit, Veracruz Institute of Technology, Calzada M. A. de Quevedo 2779, \\ 91860, Veracruz, VER., Mexico \\ ${ }^{4}$ Pathology Laboratory, Institute of Forensic Medicine, University of Veracruz, SS Juan Pablo II $s / n$, \\ 94294, Boca del Río, VER., Mexico \\ ${ }^{5}$ Biological-Chemistry Area, Veracruz Institute of Technology, Calzada M. A. de Quevedo 2779, \\ 91860, Veracruz, VER., Mexico
}

Correspondence should be addressed to Octavio Carvajal-Zarrabal; ocarvajal@uv.mx

Received 13 November 2013; Revised 16 March 2014; Accepted 17 March 2014; Published 17 April 2014

Academic Editor: Kota V. Ramana

Copyright (C) 2014 Octavio Carvajal-Zarrabal et al. This is an open access article distributed under the Creative Commons Attribution License, which permits unrestricted use, distribution, and reproduction in any medium, provided the original work is properly cited.

\begin{abstract}
Metabolic changes, along with cardiovascular and hepatic factors, are associated with the development of diseases such as diabetes, dyslipidemia, and obesity. We evaluated the effect of avocado oil supplementation (centrifuged and solvent extracted), compared with olive oil, upon the hepatic function in sucrose-fed rats. Twenty-five rats were divided into five groups: control (basal diet), a sucrose-fed group (basal diet plus 30\% sucrose solution), and three other groups (S-OO, S-AOC, and S-AOS, indicating basal diet plus 30\% sucrose solution plus olive oil OO, avocado oil extracted by centrifugation AOC or using solvent AOS, resp.). Glucose, total cholesterol, triglycerides, total protein, albumin, globulin, direct bilirubin, glutamic pyruvic transaminase, glutamic oxaloacetic transaminase, alkaline phosphatase, cholinesterase, and $\alpha$-amylase concentrations were determined and avocado oil effect on them was studied. In some cases the induced metabolic alteration significantly affected total protein and bilirubin levels and also had a highly significant effect on $\alpha$-amylase levels. AOC and AOS exhibited effects similar to those of olive oil, according to the nonsignificant difference in fatty acid profile observed by other authors. Avocado oil consumption could be beneficial in the control of altered metabolic profile illnesses as it presents effects on hepatic function biochemical markers similar to olive oil.
\end{abstract}

\section{Introduction}

The incidence of metabolic syndrome in Mexico is one of the highest in the world, so much that it has become a public health problem. However, little has been done to prevent the factors responsible for this. Epidemiological studies in our country highlight the need to strengthen strategies for its detection, control, and treatment. It involves a set of three or more alterations such as overweight or obesity and disturbance in glucose metabolism and insulin, along with hypertension, dyslipidemia, and other abnormalities of importance that are related to its development and are grouped in different profiles, such as liver, pancreatic, and cardiovascular functions [1-4]. The incidence is about $25 \%$ in the general population and there are no differences between men and women, although it varies according to genetic 
factors $[5,6]$. Reports in the scientific literature show the benefits that the Mediterranean diet and olive oil have on health $[7,8]$. These have aroused interest in studying oils rich in monounsaturated fatty acids, especially avocado oil and its effect on manifestations of health disorders in metabolic abnormality. In nonpharmacological treatment, the consumption of monounsaturated fatty acids such as oleic acid, found in different types of oils like olive and avocado, is recommended. The avocado fruit is a rich potential source of oil, mostly monounsaturated [9], and a good source of linoleic acid [10]. It also contains high levels of antioxidants including polyphenols, proanthocyanidins, tocopherols, and carotenoids, which have shown positive outcomes in health. It has also been established that soluble components of avocado oil confer these properties. Studies in humans and animal models have showed that it helps to control weight, reduces the risk of diabetes [11], normalizes blood cholesterol levels [12], and is involved in liver metabolism [13]. In addition, the phytochemical components of avocado oil are also related to the disease manifestations associated with an altered metabolic profile, so, overall, it is expected that all the beneficial properties of avocado oil together will add up to positive health effects. The purpose of this study was to evaluate the effect of avocado oil supplementation, as compared to that of olive oil, upon biochemical markers and hepatic function associated with a condition induced in rats through the administration of sucrose in drinking water.

\section{Materials and Methods}

2.1. Avocado Oil Extraction. There are different technologies for extracting oil from avocados and they can affect its quality. The oil was obtained from Hass avocado purchased from a local market in the Port of Veracruz, Mexico. When edible maturity had been reached, the avocados were washed and peeled and the seed removed. Subsequently, the pulp was homogenized by adding tert-butylhydroquinone (TBHQ) at $0.1 \%(\mathrm{w} / \mathrm{w})$.

2.1.1. Oil Extraction by Centrifugation. The avocado pulp was mixed with water to achieve a $1: 1 \mathrm{w} / \mathrm{v}$ and $\mathrm{NaCl}(7.5 \% \mathrm{w} / \mathrm{w})$, the $\mathrm{pH}$ was adjusted to 5.5 with ascorbic acid and the mixture was homogenized in a blender (Black \& Decker Model MX 150 ) at $1,300 \mathrm{rpm}$ for 1 hour at $35^{\circ} \mathrm{C}$. Subsequently, the oil was removed by centrifugation at $27000 \mathrm{rpm}$ in a tubular continuous centrifuge (Cepa-Schnell, GLE Model NBS) fed at $2.8 \mathrm{~L} / \mathrm{min}$.

2.1.2. Avocado Oil Extraction by Solvent. A homogenate was made with a portion of the avocado pulp and two parts of a mixture of hexane-isopropanol $(2: 3 \mathrm{v} / \mathrm{v})$ in separate funnels, and the oil phase was collected. Subsequently, the solvent was removed in a rotary evaporator (Buchi R-215, Labortechnik AG, Switzerland) at $30^{\circ} \mathrm{C}$ and $500 \mathrm{mmHg}$ pressure. The remaining solvent was removed by entrainment with nitrogen gas and then the oil was exposed to high vacuum in a freeze dryer for $24 \mathrm{~h}$. Thereafter the oil was stored in refrigeration and protected from light until use.
TABLE 1: Composition of basal and experimental diets formulated according to AIN-76G.

\begin{tabular}{lc}
\hline Ingredients & Basal diet $(\mathrm{g})$ \\
\hline Cornstarch & 65.8 \\
Casein & 44.0 \\
Cellulose & 4.0 \\
Mineral Mix AING-76 G & 8.0 \\
Vitamin Mix AING-76 G & 2.0 \\
DL-Methionine & 0.32 \\
Tert-butylhydroquinone & 0.02 \\
Fat $^{\dagger}$ & 10.0 \\
\hline
\end{tabular}

${ }^{\dagger}$ Corn-canola, olive, or avocado oil. Experimental diets were formulated with basal diet plus oil: olive or avocado oil extracted either by centrifugation or solvent.

TABLE 2: Fatty acid composition of dietary oils (\%).

\begin{tabular}{lccccc}
\hline Fatty acid & Corn & Canola & Olive & Avocado $^{c}$ & Avocado $^{\text {s }}$ \\
\hline $16: 0$ & 10.0 & 7.5 & 15.0 & 17.0 & 16.0 \\
$16: 1$ & 0.1 & 0.2 & 1.9 & 8.3 & 6.5 \\
$18: 0$ & 2.4 & 3.3 & 2.4 & 0.5 & 0.5 \\
$18: 1$ & 39.0 & 32.0 & 59.4 & 54.4 & 58.8 \\
$18: 2$ & 50.0 & 37.0 & 15.4 & 10.2 & 9.6 \\
$18: 3$ & 2.5 & 7.7 & 0.9 & 0.9 & 0.9 \\
\hline
\end{tabular}

Values are expressed as mean of duplicate analysis. Avocado ${ }^{c}$ : avocado oil extracted by centrifugation, Avocado ${ }^{\text {s }}$ avocado oil extracted by solvent.

2.2. Animals and Treatments. In this experiment 25 male

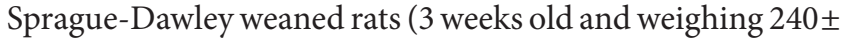
$16 \mathrm{~g}$ ) were purchased from Teklad, Co. (Mexico City), and caged individually in stainless steel boxes in a room with controlled temperature $\left(25^{\circ} \mathrm{C}\right)$ and a light-dark cycle of 12 hours. The experimental protocol for the management of experimental animals was approved by the animal ethics committee, Biochemical and Nutrition Chemistry Area, University of Veracruz. The basal diet was prepared according to the American Institute of Nutrition [14] as shown in Table 1. A mixture of corn-canola oil ( $7.5 \mathrm{~g} / 100 \mathrm{~g}$ diet) was used as a source of dietary fat (Patrona from the local market). The experimental diet was prepared based on the composition of the basal diet plus oil $(7.5 \% \mathrm{w} / \mathrm{w})$, olive oil (Carbonell), and avocado oil extracted by centrifugation or solvent, respectively. Diets were prepared once a week and kept in powder form $4^{\circ} \mathrm{C}$ until use. As part of this study, the fatty acid composition of the oils used in preparing diets was analyzed and it was found that all the oils had a rather similar composition, mainly oleic and linoleic acids (Table 2).

The animals were divided into two groups: a control group ( $\mathrm{CG}, n=5$ ) receiving a basal diet and a sucrosefed group ( $\mathrm{S}, n=20)$ which received the basal diet plus $30 \%$ sucrose solution as drinking water. The animals had free access to food and water for 16 weeks and food intake was measured daily. At the end of this period, the diet was withdrawn for at least 4 hours and the manifestation of the metabolic characteristics was checked by first determining 
body weight, then serum glucose, triglycerides, and cholesterol levels by cardiac puncture.

Once the sucrose-fed model had been obtained, the S animals were divided into four groups of five rats each. One group was maintained on the basal diet (the sucrosefed group, S); three groups of rats designated, as S-OO, SAOC, and S-AOS, respectively, received an experimental diet containing $7.5 \% \mathrm{w} / \mathrm{w}$ oil (olive and avocado extracted by centrifugation or extracted with solvent) as the sole source of dietary fat. These four groups received the experimental diets and water with $30 \%$ sucrose solution for 4 weeks. The CG group continued to receive only the diet with corncanola oil and no sucrose in the drinking water. Diets were prepared once a week and kept refrigerated until use. Tertbutylhydroquinone (TBHQ) at $0.02 \%$ was used to prevent fatty acid oxidation. At the end of the experiment, the diet was withdrawn and the fasting animals were sacrificed through decapitation. Serum glucose, cholesterol, triglyceride, and phospholipid levels were determined. All animals were sacrificed and the organs were extracted for further analysis.

2.3. Assays. Glucose was determined with the glucose oxidase method. Total cholesterol, triglycerides (TG), total protein, albumin, globulin, direct bilirubin, glutamic pyruvic transaminase (GPT), glutamic oxaloacetic transaminase (GOT), alkaline phosphatase (ALP), cholinesterase, and $\alpha$ amylase were determined with an automated analyzer (RA 1000 XT, Bayer Technicon) through enzymatic colorimetric methods using commercial kits obtained from Bayer and BioMerieux. The fatty acid profile of vegetable oils was determined through gas chromatography (Hewlett Packard 5890, Palo Alto, CA.) with pentadecanoic acid as internal standard. All the chemicals used were of analytical grade.

2.4. Statistical Analysis. The data are expressed as the mean \pm standard deviation $(x \pm \mathrm{SD})$. Statistical significance was determined with analysis of variance procedures, with a post hoc Tukey multiple-range test for comparison of means $(P<$ $0.05)$.

\section{Results}

3.1. Metabolic Characteristics of Rats in the Control and Sucrose-Fed Group. Table 3 shows growth variables, food and caloric intake, liquid consumption, and biochemical markers to assess rats in the control group (CG) and sucrose-fed rats (S). At 16 weeks, a significant increase $(P<0.05)$ in final body weight and body weight gain was observed in the $S$ group as compared to the CG group. The food intake in rats in the CG group was significantly higher $(P<0.01)$ than in the $S$ group. On the other hand, the $S$ group showed a daily liquid intake significantly higher $(P<0.05)$ as compared with the CG group. However, when the daily liquid intake per $100 \mathrm{~g}$ in weight was compared between CG and S group, this was not significant. The caloric equivalent produced by liquid intake was $10.8 \pm 1.7 \mathrm{kcal}$ in the $\mathrm{S}$ group; the CG group did not have any energy intake because this group received only purified drinking water. Triglyceride levels in the $S$ group
TABLE 3: Growth, food and caloric intake, liquid consumption, and biochemical indicators in CG and $\mathrm{S}$ rats.

\begin{tabular}{lcc}
\hline \multirow{2}{*}{ Parameters } & \multicolumn{2}{c}{ Dietary groups } \\
& CG group & S group \\
\hline Initial body weight $(\mathrm{g})$ & $239 \pm 22$ & $242 \pm 24$ \\
Final body weight $(\mathrm{g})$ & $445 \pm 53$ & $470 \pm 38^{*}$ \\
Body weight gain $(\mathrm{g})$ & $206 \pm 1.8$ & $228 \pm 2.0^{*}$ \\
Food intake (g/d) & $26.1 \pm 1.3$ & $14.3 \pm 1.1^{* *}$ \\
Liquid consumption (mL/d) & $46.3 \pm 3.3$ & $58.1 \pm 3.4^{*}$ \\
Liquid consumption (mL/d/100 g bw) & $9.3 \pm 1.4$ & $10.5 \pm 0.6$ \\
Equivalent in $\mathrm{kcal}$ in drinking water & 0.00 & $10.8 \pm 1.7^{* *}$ \\
Glucose $(\mathrm{mg} / \mathrm{dL})$ & $114 \pm 18$ & $130 \pm 11$ \\
Cholesterol $(\mathrm{mg} / \mathrm{dL})$ & $104 \pm 12$ & $101 \pm 12$ \\
Triglycerides $(\mathrm{mg} / \mathrm{dL})$ & $79 \pm 12$ & $179 \pm 35^{* *}$ \\
\hline
\end{tabular}

Values are mean \pm SD. CG group, $n=5$; group, $n=20 .{ }^{*} P<0.05 ;{ }^{* *} P<$ 0.01 .

were significantly greater $(P<0.01)$ than in the CG group; however, no significantly different results were found in any group for either glucose or cholesterol levels.

3.2. Effect of Dietary Oils on Liver Function Biochemical Markers. The effect of olive and avocado oils on liver function indicators is shown in Figure 1. S, S-OO, S-AOC, and S-AOS study groups showed changes in total protein levels, all of them significantly higher $(P<0.05)$ than control group (CG). Additionally, direct bilirubin levels in S-OO and SAOC groups decreased $(P<0.05)$ in relation to $C G$ and $S$ groups, but not for the $\mathrm{S}$-AOS group, where a nonsignificant decrease was observed. Albumin and globulin levels in SOO, S-AOC, and S-AOS study groups were not found to be significantly different either from S or CG groups.

\subsection{Effect of Dietary Oils on Pancreatic Function Biochemical} Markers. The effect of dietary olive and avocado oils on pancreatic function indicators is shown in Figure 2. Levels of glutamic oxaloacetic transaminase (GOT) in S-AOC and S-AOS groups were similar and not significantly different than either the S or CG group. Significantly lower levels were observed in the S-OO group $(P<0.05)$ in comparison with all the groups in the study. In the cases of glutamic pyruvic transaminase (GPT) and alkaline phosphatase (ALP), no significant results were observed among the study groups. The S-AOS group showed similar, nonsignificant values for cholinesterase in comparison with CG. In contrast, $S$ and $S-$ AOC groups both presented significantly lower results $(P<$ $0.05)$, but in the case of S-OO group these levels decreased in a highly significant manner $(P<0.01)$ when compared to CG and S-AOS. Levels of $\alpha$-amylase were all similar for $\mathrm{S}$, S-OO, S-AOC, and S-AOS groups; however, when compared to control group $C G$, the results were all very significantly higher $(P<0.01)$. 


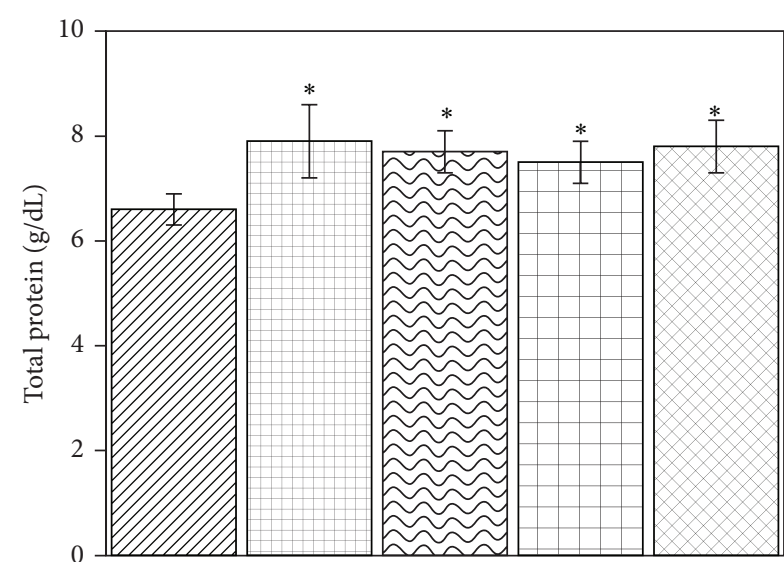

(a)

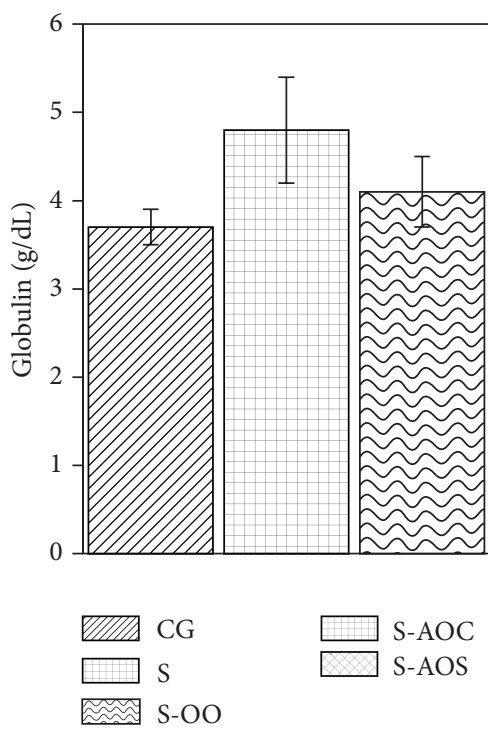

(c)

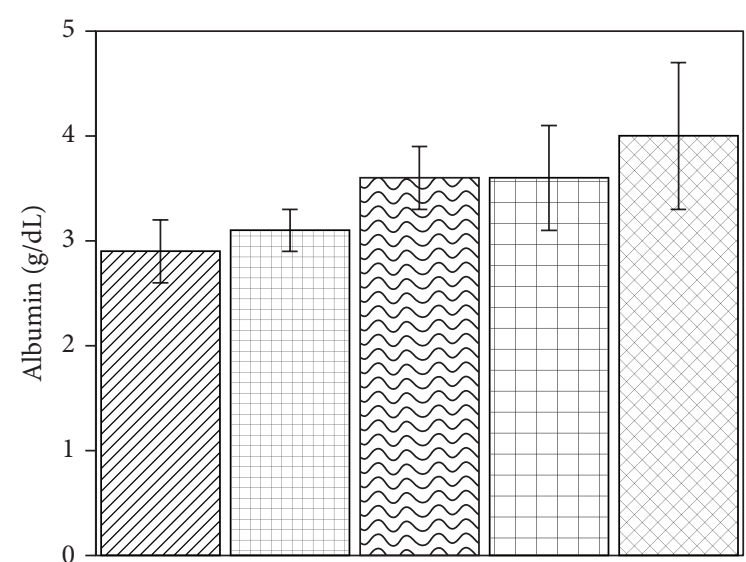

(b)

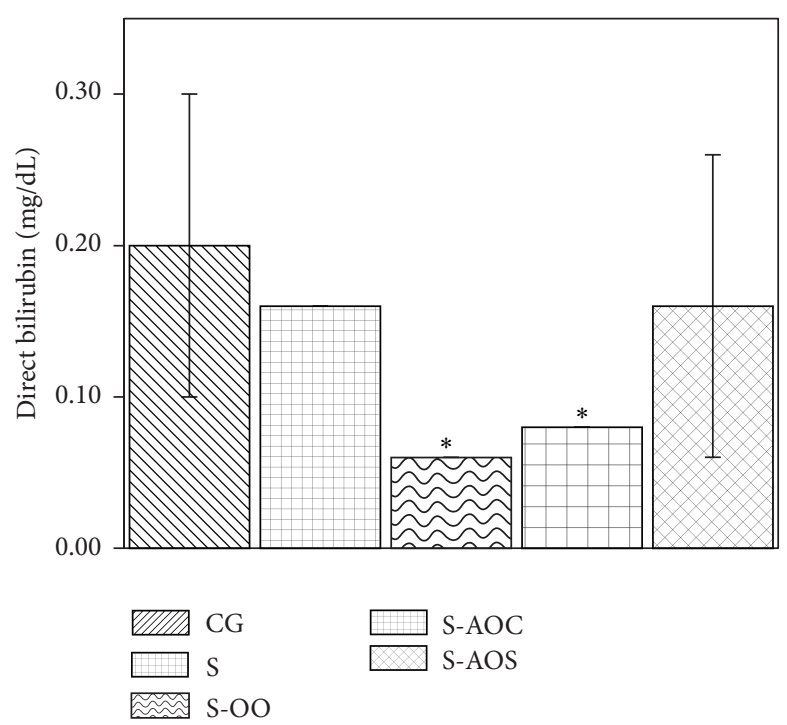

(d)

FiguRE 1: Blood serum profile levels of liver function markers in rats fed with different oil diets: (a) total protein; (b) albumin; (c) globulin; (d) direct bilirubin. Corn-canola diet plus 30\% sucrose in drinking water (S group, $n=5$ ); olive oil diet plus $30 \%$ sucrose in drinking water (S-OO group, $n=5$ ); avocado oil diet extracted by centrifugation plus $30 \%$ sucrose in drinking water (S-AOC group, $n=5$ ); avocado oil diet extracted by solvent plus $30 \%$ sucrose in drinking water (S-AOS group, $n=5$ ). Values are mean \pm SD. ${ }^{*} P<0.05 ;{ }^{* *} P<0.01$ versus corresponding data in CG group.

\section{Discussion}

Diabetes, dyslipidemia, and obesity are risk factors with a great impact on the development of diseases associated with an altered metabolic profile or metabolic syndrome. Liver and heart factors also play an important role.

Within this framework, in the present study, significant differences were found for $S$ group as compared to the CG group in final body weight and weight gain, which were significantly higher (6 and $11 \%$ resp.), although food intake was significantly lower (54\%). These results are consistent with those reported in other studies where metabolic changes were induced by the administration of a sucrose-rich diet in addition to an experimental diet causing changes in the biochemical indicators measured $[15,16]$. In relation to serum biochemical indicators associated with the development of metabolic abnormality, it was found that glucose and cholesterol concentrations in $S$ group rats were similar to those in the CG group and not significant. Reaven and Chang [17] have suggested that this is due to hyperinsulinemia developed in metabolic abnormalities which maintains normal levels of blood glucose. TG levels were significantly higher (56\%) in $S$ group rats (a 2.3-fold increase). Other studies have found similar results $[18,19]$; Piatti et al. [20] reported the association in healthy patients between sudden TG elevation and insulin resistance and suggested that the increase in blood TG in vivo inhibits glucose utilization and oxidation stimulated by insulin action in the peripheral tissues. One way to explain the blood TG elevation might be to consider a possible increase in the reesterification of fatty acids from 


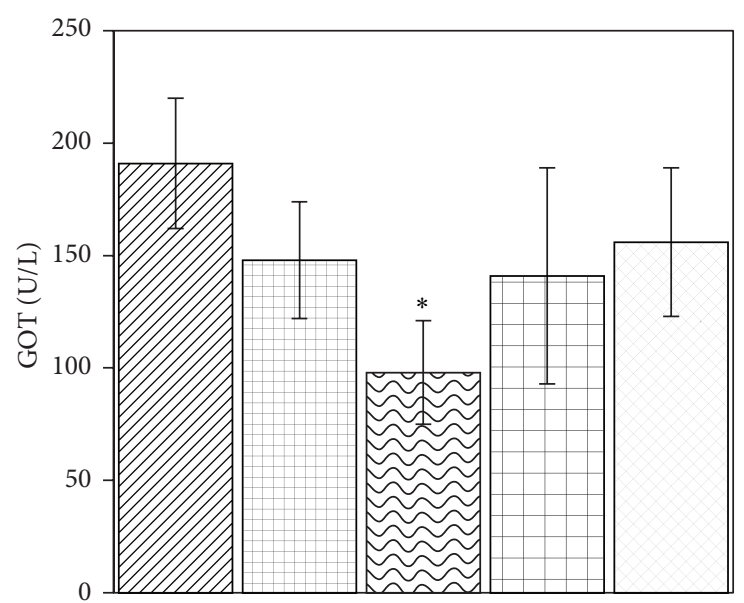

(a)

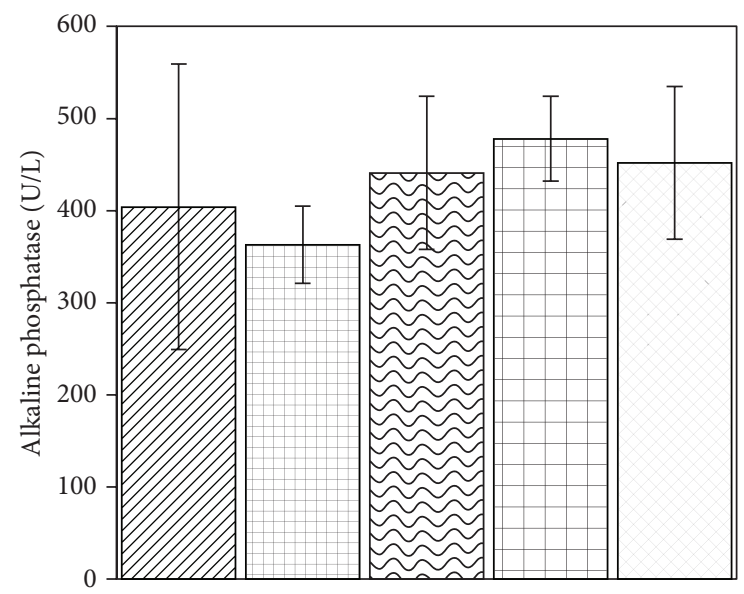

(c)

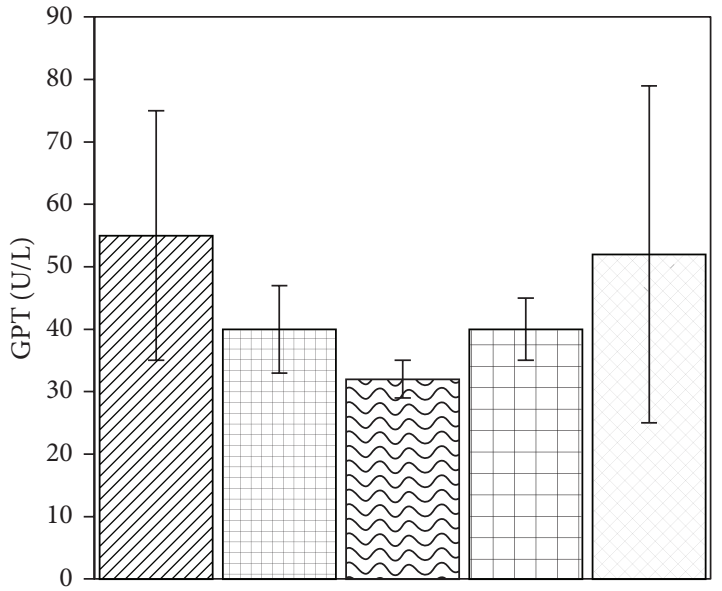

(b)

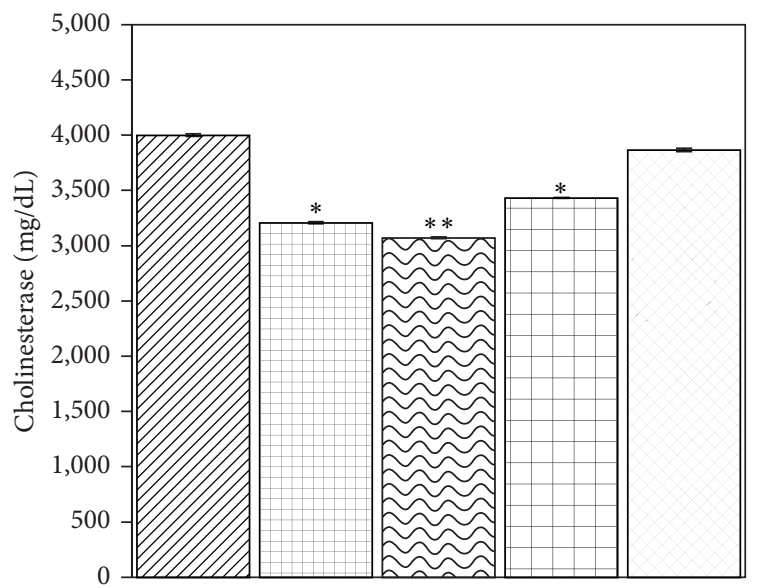

(d)

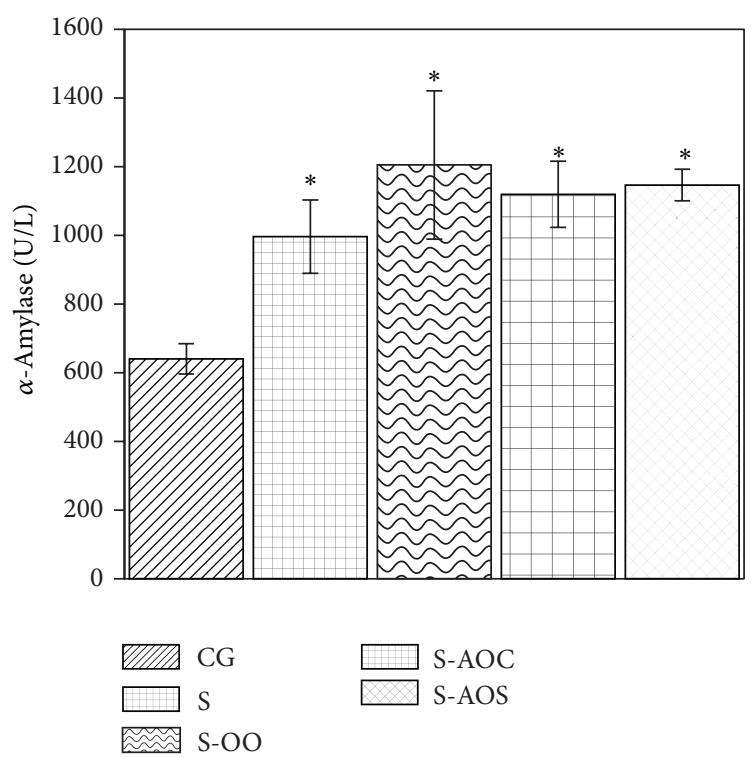

(e)

FIGURE 2: Blood serum profile levels of liver function markers in rats fed with different oil diets: (a) GOT; (b) GPT; (c) Alkaline phosphatase; (d) Cholinesterase; (e) $\alpha$-amylase. Corn-canola diet (CG group, $n=5$ ); corn-canola diet plus $30 \%$ sucrose in drinking water (S group, $n=5$ ); olive oil diet plus $30 \%$ sucrose in drinking water (S-OO group, $n=5$ ); avocado oil diet extracted by centrifugation plus $30 \%$ sucrose in drinking water (S-AOC group, $n=5$ ); avocado oil diet extracted by solvent plus $30 \%$ sucrose in drinking water (S-AOS group, $n=5$ ). GOT: glutamic oxaloacetic transaminase; GPT: glutamic pyruvic transaminase. Values are mean \pm SD. ${ }^{*} P<0.05$; ${ }^{* *} P<0.01$ versus corresponding data in CG group. 
the liver as a result of fructose metabolism as reported by Bezerra et al. [18]; this monosaccharide stems from sucrose hydrolysis and, in the liver, fatty acids are mainly used for the high-density lipoprotein (HDL) and TG synthesis, which in turn raise their serum levels.

Sucrose intake did not wield a significant influence on total protein levels as values encountered in sucrose-fed study group and in those fed with olive oil and avocado oil extracted by centrifugation or by solvents were statistically similar (7.9, 7.7, 7.5, and $7.8 \mathrm{~g} / \mathrm{dL}$, resp.) for S, S-OO, S-AOC, and S-AOS groups. Nevertheless, total protein values for the sucrose-fed group (S), as well as for S-OO, S-AOC, and S-AOS groups, increased significantly $(16,14,12$, and $15 \%$, resp.) compared to control (CG). This result suggests that avocado oil exerts an effect similar to that of olive oil on liver synthesis of total proteins which are used as markers for liver damage; a rise in these proteins is associated with the development of a nonalcoholic liver pathology, such as fatty liver, linked to metabolic syndrome [21].

Direct bilirubin indicates a loss in hepatocyte function; these levels decreased significantly in S-OO and S-AOC groups in this study compared to control (CG), sucrose-fed group (S), and the group fed with avocado oil extracted by solvent $(70,63,63 \%$ and $60,50,50 \%$, resp.). No significant differences were found for albumin and globulin levels in any group in the study. However, albumin levels in the groups fed with avocado oil extracted by centrifugation or solvent were slightly greater, although not significantly so, compared to control $(3.6,4.0$ versus $2.9 \mathrm{~g} / \mathrm{dL}$, resp.). This indicates that albumin blood levels (a liver damage marker) are not brought back to normal levels by this oil, possibly suggesting that avocado oil composition as a result of the extraction method could have an influence on the level of regulation of this marker.

On the other hand, globulin levels in S group increased (23\%), although not significantly, when compared to control. In other groups, S-OO, S-AOC, and S-AOS, globulin levels exhibited a nonsignificant decrease $(15,19$, and $21 \%$, resp.) compared to the sucrose-fed group (S) but were slightly higher, again not significantly so $(10,5$, and $3 \%$, resp.) than control (CG). These results indicate a return to normal globulin levels as a result of olive and avocado oil consumption. This could have induced a reversion to the damage caused to the liver parenchyma through steatosis, given that in this pathology blood globulin levels remain high.

It was also found that albumin/globulin ratio values (RAG) of rats fed olive oil and avocado oil extracted by centrifugation or by solvents were higher than the sucrosefed group $(0.9,0.9$, and 1.0 versus 0.7 , resp.). The results for this marker could suggest a regeneration of liver tissue, as RAG values of the aforementioned study groups were close to 1 , similar to the CG value. S group, on the other hand, had quite a low value for this ratio which suggests liver damage.

Mention should be made that to date no reports have appeared in the scientific literature about the effect of avocado oil on the basic profile of protein synthesized by the liver which are used as liver damage markers, specifically cirrhosis of the liver and fatty liver, thus warranting the determination of these values in the present study.
As for control group (CG), GOT and GPT values decreased in the rest of the groups (S, S-OO, S-AOC and $\mathrm{S}$-AOS). An increase, not a decrease, in these enzymes is indicative of liver failure. The effect of administering a diet rich in carbohydrates ( $30 \%$ sucrose), known to contribute to metabolic syndrome in murine models through oxidative stress, or through the activation of adipocyte-specific genes (lipogenesis), among other effects, and in consequence to hepatic metabolic changes [22-24] was not observed in this case related to an increase in GOT and GPT values.

A significant decrease in GOT was observed in the SOO group. Both olive and avocado oils are recognized as oils with a high percentage of unsaturated fatty acids and a low percentage of saturated $[25,26],(84,88$ and $17,12 \%$, resp.) in murine models, different transcriptomic responses between diets based on different long-chain polyunsaturated fatty acids have been observed. Furthermore, stereochemistry influences differential responses as seen with linoleic acid isomers [23].

Sucrose intake significantly decreased cholinesterase levels in the sucrose-fed group (S) by $20 \%$ compared to control. Olive oil did not improve this situation at all and in fact the results of the S-OO group were significantly even lower than S. Avocado oil extracted by solvent brought cholinesterase levels back to normal, similar to those of control (3866 versus $3999 \mathrm{mg} / \mathrm{dL}$ resp.), but, for the S-AOC group, results remained at the same level statistically as the sucrose-fed group (S).

A decrease in cholinesterase levels can be indicative of hepatic disease. As far as can be ascertained, the different responses of olive and avocado oil observed here have not been reported before. A possible explanation could be based on the specific chemical composition of each oil type and their capacity to overcome the diet induced metabolic alterations. The unsaponifiable fraction of fatty oils contains more than 100 components. A specific example is the case of olive oil whose fraction contains more than 300 components [27]. It has been demonstrated that these minor components also exert effects on the metabolic pathways and according to Osada, 2013, the term "monounsaturated fatty acid-enriched oil" including oils such as olive and other oils, based on the high percentage of oleic acid, "no longer appears appropriate for describing their biological properties since they have different unsaponifiable composition and this fraction is highly active."

For S, S-OO, S-AOC, and S-AOS groups in the study, $\alpha$ amylase levels were found to be significantly higher compared to control $(36,47,43$, and $44 \%$, resp.). This points to pancreas damage which was not reversed by the administration of dietary oils (S-OO, S-AOC, and S-AOS); in these groups, significantly higher $\alpha$-amylase levels were found $(1205,1119$, and 1146 versus $996 \mathrm{U} / \mathrm{L}$, resp.) compared to S. To our knowledge, no data are available in the literature about the effect of avocado oil on the levels of this enzyme, even less where sucrose ingestion is concerned.

Sucrose intake caused no effect in ALP levels in SOO, S-AOC, and S-AOS groups as these were similar and did not differ from control (CG) or sucrose-fed group (S). However, ALP levels in S-AOC and S-AOS groups were seen 
to increase (15 and $11 \%$, resp.) in comparison with control and 24 and $20 \%$, respectively, when compared to S. It has been reported that in cases of nonalcoholic fatty liver disease alkaline phosphatase levels increase and are a favorite marker for metabolic syndrome [28].

\section{Conclusion}

To sum up, these results indicate that sucrose intake affected total protein and bilirubin levels; the results of other markers did not show any evidence of liver damage, probably because the window for manifestation was very short. However, variations in the aforementioned marker levels indicate that the liver was affected and that the albumin/globulin ratio (RAG) under the influence of avocado oil administration revealed the beginning of a regeneration of liver function. On the other hand, neither the administration of olive oil nor avocado oil extracted by centrifugation or using solvent was able to attain normal $\alpha$-amylase levels, indicating that anomalies in pancreatic function were not reversed. Avocado oil exhibits effects similar to those of olive oil, and no differences in biochemical markers were found between the two methods of avocado oil extraction. This finding is correlated to the nonsignificant difference observed in fatty acid profile of avocado oils obtained by the two aforementioned extraction methods, as reported by Ariza-Ortega et al. [29].

\section{Conflict of Interests}

The authors have declared that no competing interest exists.

\section{References}

[1] S. D. H. Malnick, M. Beergabel, and H. Knobler, "Non-alcoholic fatty liver: a common manifestation of a metabolic disorder," Quarterly Journal of Medicine, vol. 96, no. 10, pp. 699-709, 2003.

[2] Z. Gong and R. H. Muzumdar, "Pancreatic function, type 2 diabetes, and metabolism in aging," International Journal of Endocrinology, vol. 2012, Article ID 320482, 13 pages, 2012.

[3] D.-C. Lee, X. Sui, T. S. Church, C. J. Lavie, A. S. Jackson, and S. N. Blair, "Changes in fitness and fatness on the development of cardiovascular disease risk factors: hypertension, metabolic syndrome, and hypercholesterolemia," Journal of the American College of Cardiology, vol. 59, no. 7, pp. 665-672, 2012.

[4] H. Basciano, L. Federico, and K. Adeli, "Fructose, insulin resistance, and metabolic dyslipidemia," Nutrition \& Metabolism, vol. 2, article 5, 2005.

[5] J. Carranza-Madrigal and S. M. López-Correa, "El Síndrome Metabólico en México," Internal Medicine of México, vol. 24, no. 4, pp. 251-261, 2008.

[6] G. Riccardi, R. Giacco, and A. A. Rivellese, "Dietary fat, insulin sensitivity and the metabolic syndrome," Clinical Nutrition, vol. 23, no. 4, pp. 447-456, 2004.

[7] D. B. Panagiotakos and E. Polychronopoulos, "The role of Mediterranean diet in the epidemiology of metabolic syndrome; converting epidemiology to clinical practice," Lipids in Health and Disease, vol. 4, no. 7, pp. 1-6, 2005.

[8] G. Brucker, "Fatty acids in foods and cardiovascular disease," in Fatty Acids in Food and Their Health Implications, C. K. Chow, Ed., pp. 735-752, Marcel Dekker, New York, NY, USA, 1992.
[9] R. B. H. Willis, J. S. K. Lim, and H. Greenfield, "Composition of Australian foods: tropical and subtropical fruit," Food Technology in Australia, vol. 38, pp. 118-123, 1986.

[10] B. Bergh, "Nutritious value of avocado," California Avocado Society Yearbook, vol. 76, pp. 123-135, 1992.

[11] I. Lerman-Garber, S. Ichazo-Cerro, J. Zamora-González, G. Cardoso-Saldaña, and C. Posadas-Romero, "Effect of a highmonounsaturated fat diet enriched with avocado in NIDDM patients," Diabetes Care, vol. 17, no. 4, pp. 311-315, 1994.

[12] D. Kritchevsky, S. A. Tepper, S. Wright, S. K. Czarnecki, T. A. Wilson, and R. J. Nicolosi, "Cholesterol vehicle in experimental atherosclerosis 24: avocado oil," Journal of the American College of Nutrition, vol. 22, no. 1, pp. 52-55, 2003.

[13] M. J. Werman, I. Neeman, and S. Mokady, "Avocado oils and hepatic lipid metabolism in growing rats," Food and Chemical Toxicology, vol. 29, no. 2, pp. 93-99, 1991.

[14] P. G. Reeves, F. H. Nielsen, and G. C. Fahey Jr., "AIN-93 purified diets for laboratory rodents: final report of the American Institute of Nutrition ad hoc writing committee on the reformulation of the AIN-76A rodent diet," Journal of Nutrition, vol. 123, no. 11, pp. 1939-1951, 1993.

[15] R. M. Oliart Ros, M. E. Torres-Márquez, A. Badillo, and O. Angulo Guerrero, "Dietary fatty acids effects on sucroseinduced cardiovascular syndrome in rats," Journal of Nutritional Biochemistry, vol. 12, no. 4, pp. 207-212, 2001.

[16] M. El Hafidi, A. Cuéllar, J. Ramírez, and G. Baos, "Effect of sucrose addition to drinking water, that induces hypertension in the rats, on liver microsomal $\Delta 9$ and $\Delta 5$-desaturase activities," Journal of Nutritional Biochemistry, vol. 12, no. 7, pp. 396-403, 2001.

[17] G. M. Reaven and H. Chang, "Relationship between blood pressure, plasma insulin and triglyceride concentration, and insulin action in spontaneous hypertensive and Wistar-Kyoto rats," American Journal of Hypertension, vol. 4, no. 1, pp. 34-38, 1991.

[18] R. M. N. Bezerra, M. Ueno, M. S. Silva, D. Q. Tavares, C. R. O. Carvalho, and M. J. A. Saad, "A high fructose diet affects the early steps of insulin action in muscle and liver of rats," Journal of Nutrition, vol. 130, no. 6, pp. 1531-1535, 2000.

[19] A. W. Thorburn, L. H. Storlien, A. B. Jenkins, S. Khouri, and E. W. Kraegen, "Fructose-induced in vivo insulin resistance and elevated plasma triglyceride levels in rats," American Journal of Clinical Nutrition, vol. 49, no. 6, pp. 1155-1163, 1989.

[20] P. M. Piatti, L. D. Monti, L. Baruffaldi et al., "Effects of an acute increase in plasma triglyceride levels on glucose metabolism in man," Metabolism: Clinical and Experimental, vol. 44, no. 7, pp. 883-889, 1995.

[21] J. M. Clark and A. M. Diehl, "Nonalcoholic fatty liver disease: an underrecognized cause of cryptogenic cirrhosis," Journal of the American Medical Association, vol. 289, no. 22, pp. 3000-3004, 2003.

[22] Z. H. Yang, H. Miyahara, J. Takeo, and M. Katayama, "Diet high in fat and sucrose induces rapid onset of obesity-related metabolic syndrome partly through rapid response of genes involved in lipogenesis, insulin signalling and inflammation in mice," Diabetology \& Metabolic Syndrome, vol. 4, article 32, 2012.

[23] J. Osada, "The use of transcriptomic to unveil the role of nutrients in mammalian liver," ISRN Nutrition, vol. 2013, Article ID 403792, 19 pages, 2013.

[24] E. L. B. Novelli, Nutrição e vida saudável: Estresse Oxidativo e metabolismo energético, Tecmedd Editora, São Paulo, Brazil, 2005. 
[25] R. Mailer, "Chemistry and quality of olive oil," PRIMEFACT 227, New South Wales Department of Primary Industries, 2006, http://www.dpi.nsw.gov.au/__data/assets/pdf_file/0003/87168/ pf227-Chemistry-and-quality-of-olive-oil.pdf.

[26] M. Wong, C. Requejo-Jacman, and A. Woolf, "What is unrefined, extra virgin cold-pressed avocado oil? American Oil Chemists Society AOCS," 2010 http://www.aocs.org/ Membership/FreeCover.cfm?ItemNumber=1099.

[27] J. M. Lou-Bonafonte, C. Arnal, M. A. Navarro, and J. Osada, "Efficacy of bioactive compounds from extra virgin olive oil to modulate atherosclerosis development," Molecular Nutrition and Food Research, vol. 56, no. 7, pp. 1043-1057, 2012.

[28] A. J. G. Hanley, K. Williams, A. Festa, L. E. Wagenknecht, R. B. D’Agostino Jr., and S. M. Haffner, "Liver markers and development of the metabolic syndrome: the Insulin Resistance Atherosclerosis Study," Diabetes, vol. 54, no. 11, pp. 3140-3147, 2005.

[29] J. A. Ariza-Ortega, F. Lopéz-Valdez, J. Coyotl-Huerta, M. E. Ramos-Cassellis, J. Díaz-Reyes, and A. Martínez-Zavala, "Efecto de diferentes métodos de extracción sobre el perfil de ácidos grasos de aguacate (Persea americana Mill. Var. Hass)," Revista Venezolana de Ciencia y Tecnología de Alimentos, vol. 2, no. 2, pp. 263-276, 2011. 

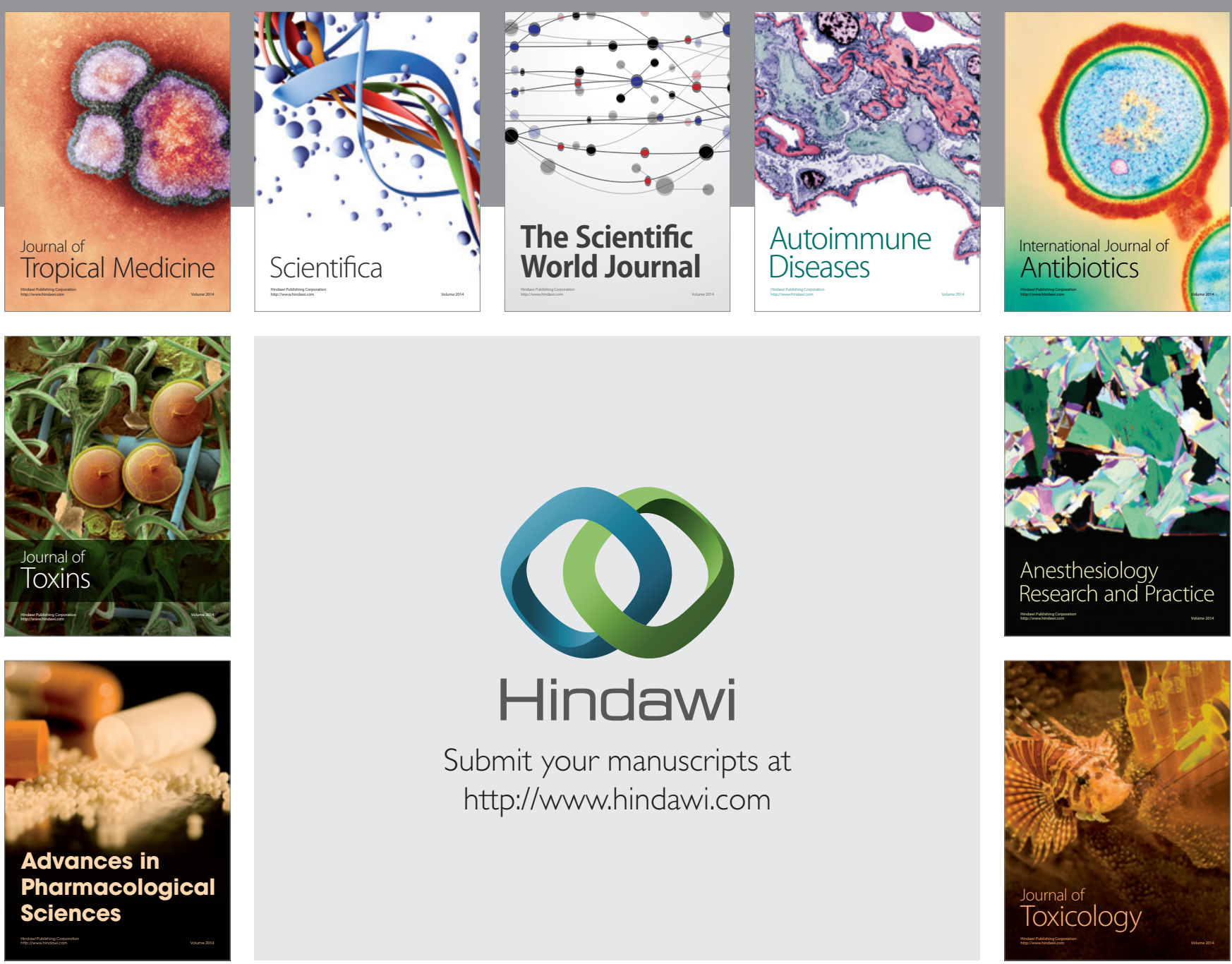

\section{Hindawi}

Submit your manuscripts at

http://www.hindawi.com
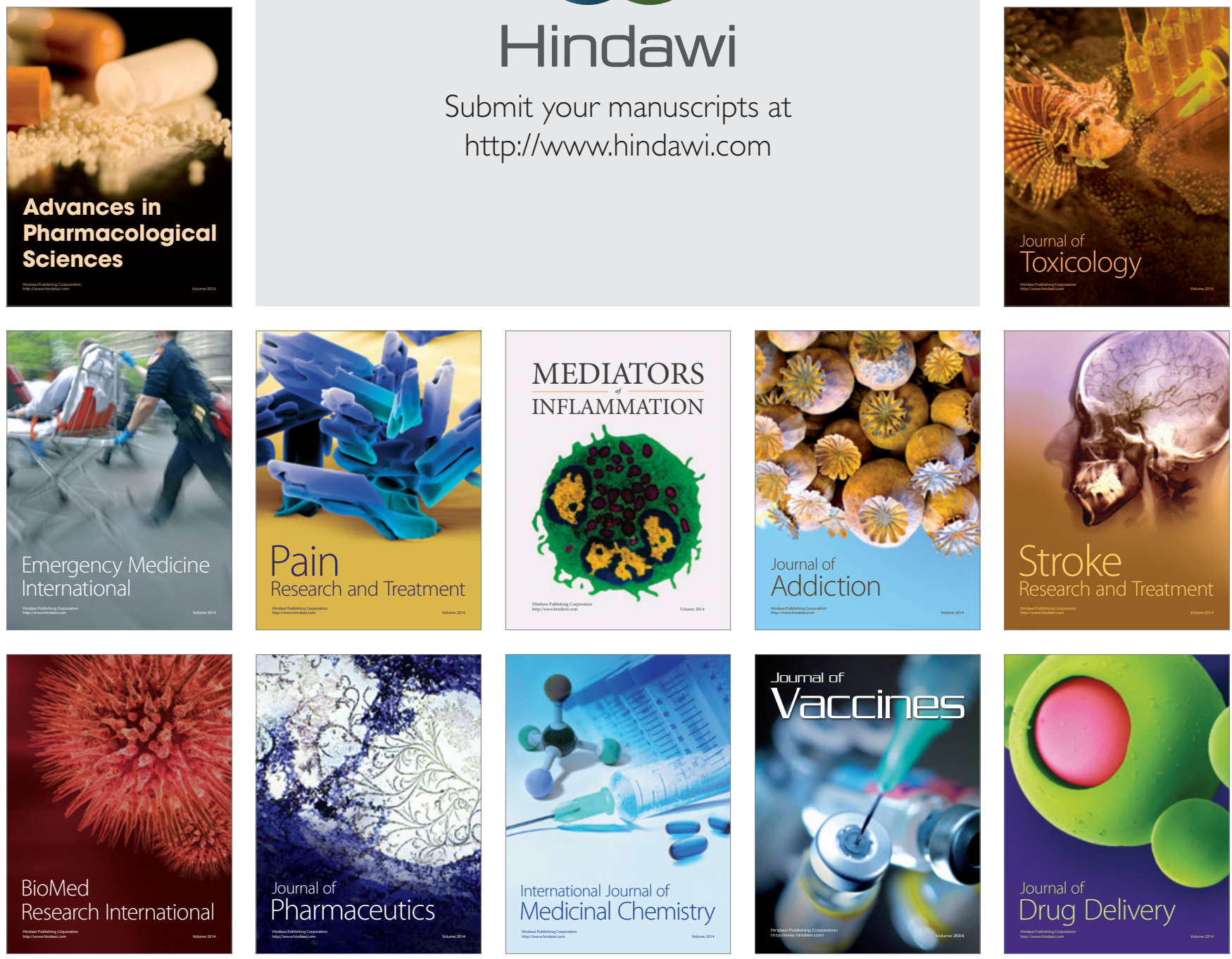